\title{
Effects of Electrode Reversal on the Distribution of Naproxen in the Electrospun Cellulose Acetate Nanofibers
}

\author{
Zhuang Li, ${ }^{1,2}$ Hongliang Kang, ${ }^{1}$ Ning Che, ${ }^{1,2}$ Zhijing Liu, ${ }^{1,2}$ Pingping Li, ${ }^{1,2}$ Weiwei Li, ${ }^{1}$ \\ Chao Zhang, ${ }^{1,2}$ Chun Cao, ${ }^{1,2}$ Ruigang Liu, ${ }^{1}$ and Yong Huang ${ }^{1,3}$ \\ ${ }^{1}$ State Key Laboratory of Polymer Physics and Chemistry, Beijing National Laboratory of Molecular Sciences, \\ Institute of Chemistry, Chinese Academy of Sciences, Beijing 100190, China \\ ${ }^{2}$ University of Chinese Academy of Sciences, Beijing 100049, China \\ ${ }^{3}$ National Research Center for Engineering Plastics, Technical Institute of Physics \& Chemistry, Chinese Academy of Sciences, \\ Beijing 100190, China
}

Correspondence should be addressed to Ruigang Liu; rgliu@iccas.ac.cn and Yong Huang; yhuang@mail.ipc.ac.cn

Received 8 January 2014; Revised 26 March 2014; Accepted 31 March 2014; Published 4 May 2014

Academic Editor: Huarong Nie

Copyright (C) 2014 Zhuang Li et al. This is an open access article distributed under the Creative Commons Attribution License, which permits unrestricted use, distribution, and reproduction in any medium, provided the original work is properly cited.

Naproxen (NAP)/cellulose acetate hybrid nanofibers were prepared by positive and reversed emitting electrodes electrospinning setups. The morphology and structure of the resultant nanofibers were characterized, and the NAP release behaviors were investigated. It was found that NAP dispersed in the CA matrix in molecular level, and no aggregation and dimers of NAP were found in the resultant NAP/CA hybrid nanofibers due to the formation of hydrogen bonds between NAP and CA. The nanofibers obtained by reversed emitting electrode electrospinning setup have a thicker diameter and a faster NAP release rate compared with those obtained by positive emitting electrode electrospinning setup. The faster drug release of NAP from nanofibers prepared by reversed emitting electrode electrospinning is due to the fact that the concentration of NAP molecules near the surface of the nanofibers is relatively higher than that of the nanofibers prepared by positive emitting electrode electrospinning setup. The effects of the electrode polarity on the distribution of drugs in nanofibers can be used to prepare hybrid electrospun fibers of different drug release rates, which may found applications in biomedical materials.

\section{Introduction}

Electrospinning is a cost effective and straightforward technique to produce nanofibers with diameters in the range of $50 \mathrm{~nm}$ to $5 \mu \mathrm{m}[1,2]$. The resultant nanofiber mats are attractive materials for their superior specific surface area and high porosity, which have promising applications in filter [36], catalyst [7-9], sensor [10-12], and biomedical materials [13-15]. In the process of electrospinning, a continuous charged jet is initiated when the electric field force exerted on the pendent drop exceeds its own surface tension. The flying jet then undergoes straight movement and whipping motion and finally solidifies on the collector as a nonwoven mat $[16,17]$. Due to the complexity of electrohydrodynamics, the process of electrospinning was affected by various factors including solution properties, experimental parameters, and environmental conditions $[18,19]$. Among various process parameters, volumetric charge density, distance between nozzle and collector, initial jet radius, relaxation time, and solution viscosity have the most significant effects on the flying jet [20]. The polarity of the emitting electrode, say the one that is in contact with the polymer solution or melt, can be either positive or negative in electrospinning. Generally, the emitting electrode is positive. Meanwhile, electrospinning with negative emitting electrode has also been applied for preparing nanofibers, and the effects of the electric field morphology on the resultant nanofibers have been investigated [21-23]. It was found that the prepared nanofibers usually have larger diameter and pore size in the case of using negative emitting electrode, which is attributed to the relatively small Coulombic repulsion force on the flying jets [24]. 


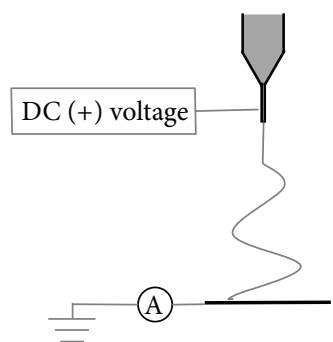

(a)

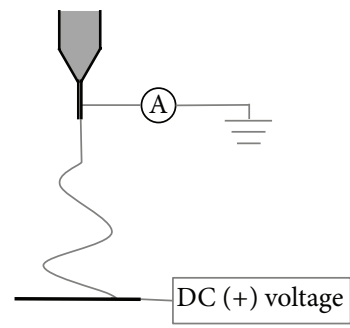

(b)
FIGURE 1: Schematic illustration of the electrospinning setup with (a) positive and (b) reversed emitting electrodes.

Due to the similarity to extracellular matrix (ECM), electrospun nanofibers loading various drugs and biomolecules have been used as wound dressing, surgical scaffold, and orthopedic materials [25-27]. The conventional method of producing these composite nanofibers was directly electrospinning the mixed solution of polymers and contents. It is reported that the loaded drugs were embedded in the polymer fibers and diffused out with a burst release in in vitro dissolution test, which is due to the interaction between the drugs and the polymer molecules [28, 29]. Naproxen (NAP) as an effective nonsteroidal anti-inflammatory drug is commonly used to treat the inflammation and pain of various wounds [30]. Owing to gastrointestinal irritancy caused by oral administration, NAP is more appropriate for topical application [31].

In this work, cellulose acetated (CA) nanofibers loaded with naproxen were prepared by electrospinning with positive and reversed emitting electrodes setups. CA and NAP mixed solution was used in the electrospinning. The morphology of the resultant nanofibers and the drug distribution were investigated and discussed.

\section{Materials and Methods}

2.1. Materials. Cellulose acetate $\left(\mathrm{CA}, M_{r}=29 \mathrm{kDa}\right)$ was purchased from Sigma Aldrich (USA). Naproxen (NAP) was purchased from Alfa Aesar (USA). Highly purified water was prepared using Milli-Q Water Purification System (Millipore, USA). Dimethyl sulfoxide (DMSO) was supplied by Beijing Chemical Works and distilled before use. Other chemicals including acetone and $N, N$-dimethylacetamide (DMAc) are all of analytical grade and were used as received.

2.2. Preparation of NAP-Loaded Nanofibers. $19 \%(\mathrm{w} / \mathrm{v}) \mathrm{CA}$ solution was prepared by dissolving CA in a mixed solution of acetone and DMAc $(2: 1, \mathrm{v} / \mathrm{v})$. Then, $10 \mathrm{wt} \%$ NAP (based on the weight of CA) was added into the base CA solution to get the mixed solution for electrospinning. The conductivity of the CA solution and the NAP/CA mixed solution was measured using a conductivity meter (Rex DDS-307A, INESA, China).

Two setups with inverse emitting electrode polarity, say positive and reversed emitting electrodes, were used for electrospinning (Figure 1). The other parameters were the same for the two setups. The inner diameter of the needle was $1.0 \mathrm{~mm}$ and the extruding rate was $0.25 \mathrm{~mL} / \mathrm{h}$. The applied voltage was $15 \mathrm{kV}$ and the distance between the needle and the collector plate was $12 \mathrm{~cm}$. All the electrospinning experiments were carried out at room temperature with a humidity of about $20-30 \%$. The electric current of both electrospinning setups was determined using a multimeter (Fluke 15B, USA).

2.3. Instruments and Characterization. The morphology of the prepared fibers was observed on a field emission scanning electron microscopy (FESEM, JEOL 6700, Japan) operated at an accelerating voltage of $5 \mathrm{kV}$ and current of $10 \mu \mathrm{A}$. The samples were sputter-coated with a layer of platinum using a sputter-coater (Bal-Tec, SCD 500, Switzerland) before observation. All the images were taken in the secondary electron mode. Elemental analysis was performed on an energy dispersive X-ray spectrometer (EDS, EDAX-TSL, USA). Transmission electron microscopy (TEM) observation of the nanofibers was carried out on a field emission transmission electron microscopy (FETEM, 2200FS, JEOL, Japan). The samples were prepared by fixing copper grids on the collector and collecting fibers during the process of electrospinning. The operation voltage of TEM was set to $200 \mathrm{kV}$, and the images were recorded in the bright field mode.

Differential scanning calorimetry (DSC) experiments were carried out on DSC Q2000 (TA Instruments, USA) in the range of $20-200^{\circ} \mathrm{C}$ at the heating rate of $10^{\circ} \mathrm{C} / \mathrm{min}$ in nitrogen atmosphere.

Wide angle X-ray diffraction (WAXD) experiments were carried out on a D/max $2500 \mathrm{X}$-ray diffraction meter (Rigaku, Japan). CuK $\alpha$ X-ray $(\lambda=0.154 \mathrm{~nm})$ was used as the radiation source. Operation parameters were set at $40 \mathrm{kV}$ and $200 \mathrm{~mA}$ and the data in the range of $2 \theta=5-60^{\circ}$ were recorded.

Fourier transform infrared spectroscopy (FTIR, EQUINOX55, Bruker, Germany) was used to characterize the fiber mats at a range of $400 \mathrm{~cm}^{-1}$ to $4000 \mathrm{~cm}^{-1}$ and a resolution of $2 \mathrm{~cm}^{-1}$.

UV-Vis spectrophotometer (TU-1901, Purkinje General, China) was used to determine the NAP concentration in the buffer solutions. The wavelength of the emitting UV-Vis light was selected as $262 \mathrm{~nm}$.

2.4. In Vitro Drug Release. The in vitro drug release experiments of both NAP/CA nanofibers were carried out as follows. A certain amount of nanofibers were placed in a dialysis bag with molecular weight cutoff of $3.50 \mathrm{kDa}$. The dialysis bag was then immersed in a phosphate buffer solution with a desired volume. The temperature of the phosphate buffer solution ( $\mathrm{pH}=6.4$ ) was set at $37 \pm 0.5^{\circ} \mathrm{C}$ to mimic the physiological conditions of human skin. The out medium was stirred continuously at $200 \mathrm{rpm}$ and the NAP concentration in the out buffer solution was determined at desired time by UV-Vis absorbance. 

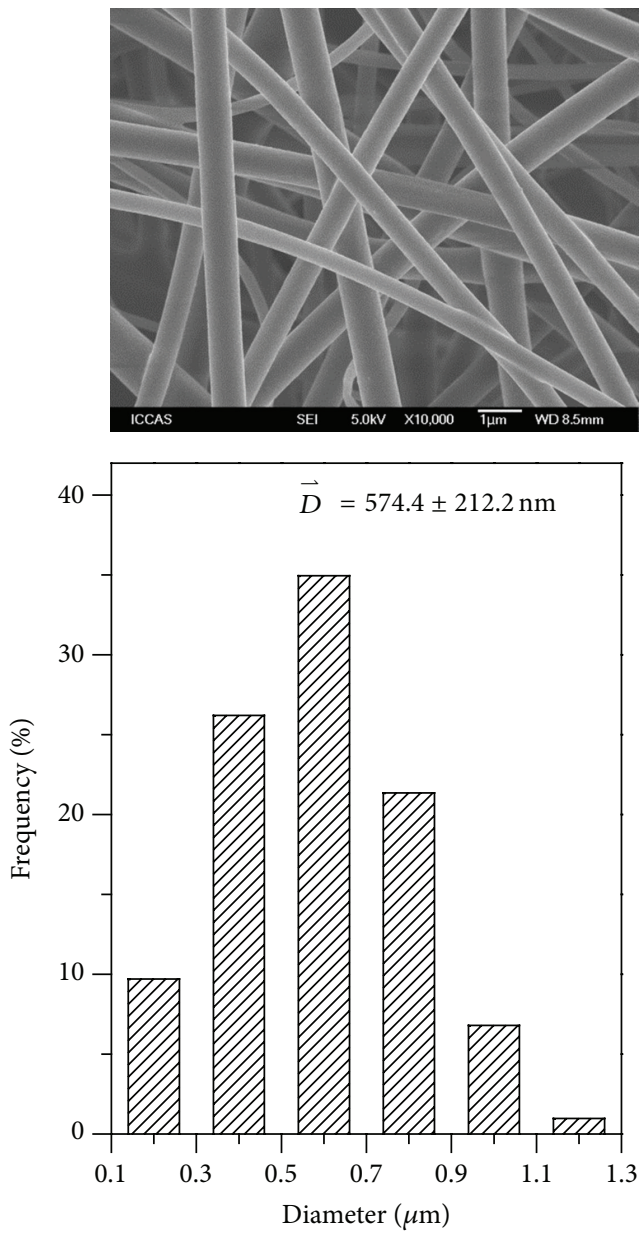

(a)
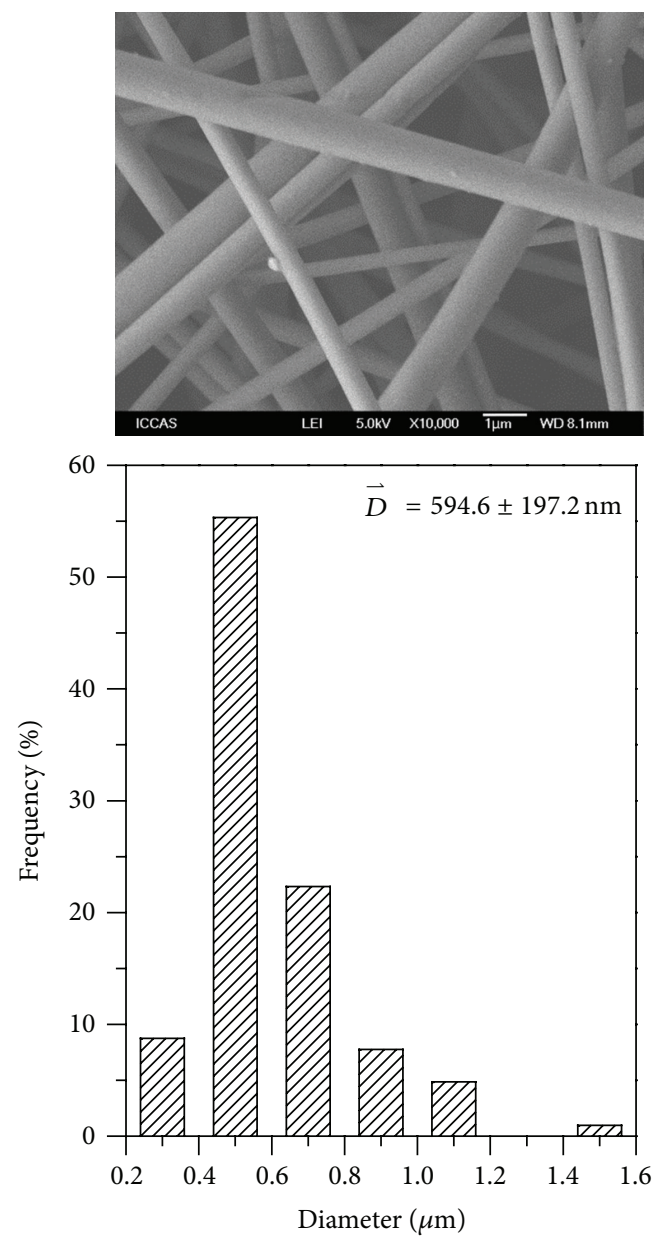

(b)

FIGURE 2: Typical SEM images and corresponding diameter distribution of the nanofibers prepared by (a) positive and (b) reversed emitting electrodes electrospinning setups.

The accumulative release of NAP from the nanofibers, $P(\%)$, was calculated using the following equation [32]:

$$
P(\%)=\frac{C_{n} \times V_{0}+\sum_{i=1}^{n-1} C_{i} \times V}{W}
$$

where $C_{n}$ is the NAP concentration determined at number $n$ and $C_{i}$ is the NAP concentration determined at number $i$; $V_{0}$ is the volume of the buffer and $V$ is the volume of the withdrawn sample; and $W$ is the total amount of NAP in the fibers. Three parallel experiments were carried out and the mean value was plotted as a function of time.

\section{Results and Discussion}

3.1. Preparation of NAP/CA Nanofibers. NAP/CA hybrid nanofiber mats can be successfully prepared by both positive and reversed emitting electrode setups (Figure S1); see Figure S1 in Supplementary Material available online at http://dx.doi.org/10.1155/2014/360658. Figure 2 shows the typical SEM images of the NAP/CA nanofibers prepared by electrospinning using positive and reversed emitting electrodes. The results indicate that the resultant nanofibers have similar morphology. The nanofibers have smooth surfaces and no particles can be observed, suggesting no NAP aggregations formed outside of the hybrid nanofibers. Statistics of the diameter of the nanofibers indicates that the nanofibers prepared by reversed emitting electrode electrospinning have a larger average diameter and a narrower diameter distribution $(594.6 \pm 197.2 \mathrm{~nm})$ compared with those nanofibers prepared by using positive emitting electrode setup (574.4 \pm $212.2 \mathrm{~nm}$ ) (Figure 2). The difference could come from the difference in the working electric current for the positive and reversed emitting electrodes electrospinning setups. It was noted that the electric current of the jets for the reversed emitting electrode approach is about $0.3-0.5 \mu \mathrm{A}$ much lower than that of the positive emitting electrode approach (4.6$5.4 \mu \mathrm{A}$ ). A lower working electric current corresponds to a lower charge density of the jet flow during electrospinning [33-35]. As a result, the electric field force stretching the jet as well as the electrostatic repulsion between the jets is accordingly smaller, leading to the relatively low stretching 


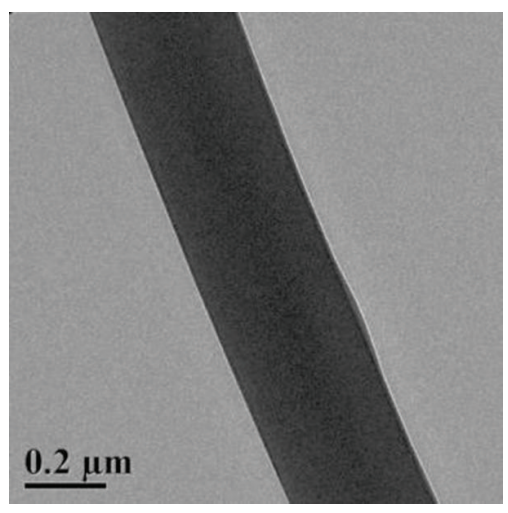

(a)

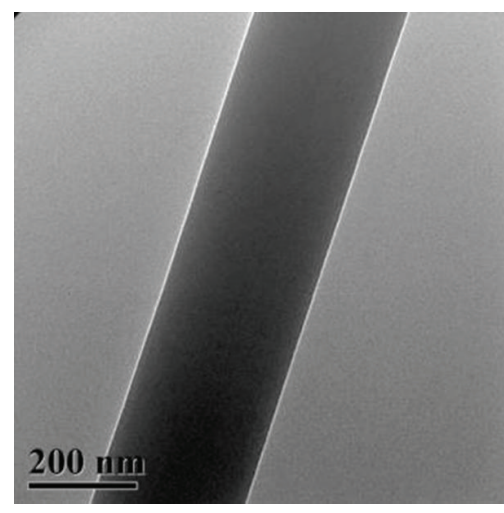

(b)

FIGURE 3: TEM images of nanofibers prepared by (a) positive and (b) reversed emitting electrodes electrospinning setups.

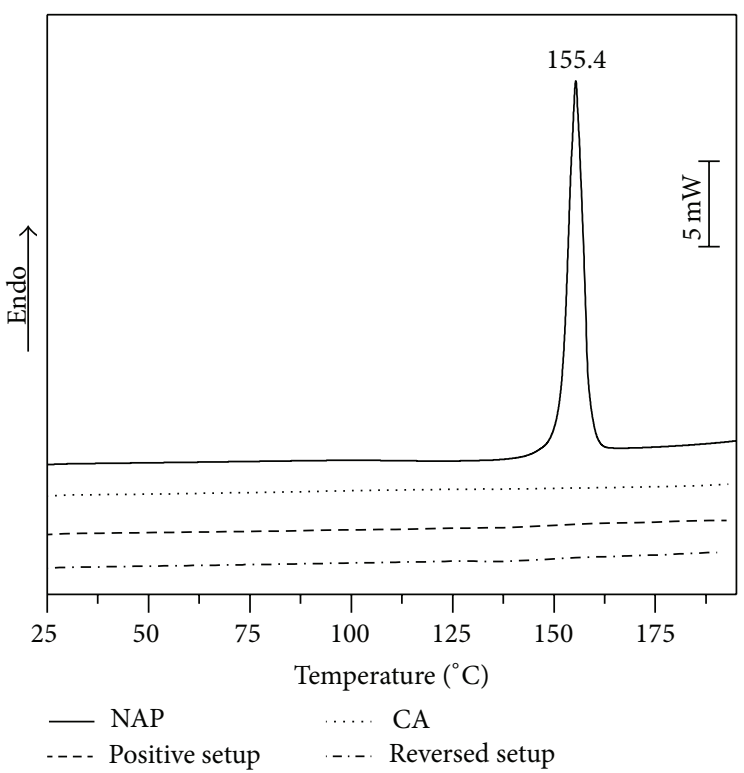

(a)

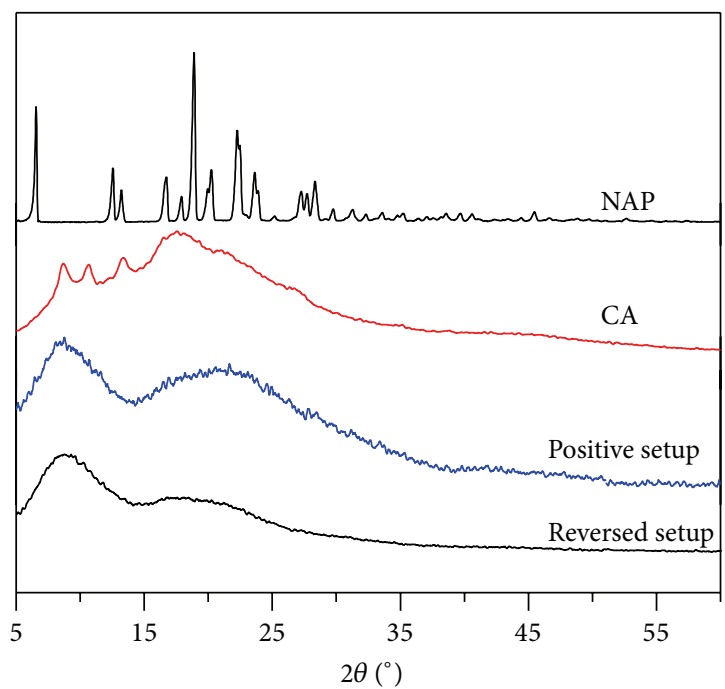

(b)

FIGURE 4: DSC traces (a) and XRD curves (b) of the NAP/CA nanofibers and correlated raw materials.

force on the jet and the thick diameter of the resultant nanofibers. TEM observation indicates that the nanofibers prepared by both positive and reversed emitting electrodes electrospinning setups have uniform structure (Figure 3), which suggests that NAP molecules are homogeneously dispersed in the CA matrixes.

3.2. Dispersion of the NAP in the CA Matrix. The solubility of hydrophobic drugs, such as NAP, is quite important for the improvement of the drug efficiency, which can be achieved by adding additives to reduce the crystallization of the drugs. Figure 4 shows the DSC and XRD curves of the NAP/CA hybrid nanofibers prepared by positive and reversed emitting electrodes electrospinning setups. Data of NAP and CA are also provided for comparison. The DSC results indicate that there is no melting peak of NAP crystal appearing on both NAP/CA nanofibers, suggesting no NAP crystal formed in either of the nanofibers. The XRD results further confirmed that no NAP crystal exists in the nanofibers. These results are consistent with the TEM observations (Figure 3), in which no NAP crystal or aggregation can be observed.

For clarification of the interactions between the NAP and the CA matrix, FTIR experiments were carried out and the results are shown in Figure 5. The well-defined sharp absorption peak of pure NAP is at $1728 \mathrm{~cm}^{-1}$, which comes from the stretching vibration of the carbonyl groups. The absorption peak of carbonyl groups of pure CA locates at $1753 \mathrm{~cm}^{-1}$. For both hybrid nanofibers, the absorption peak of carbonyl groups locates at $1743 \mathrm{~cm}^{-1}$, indicating the interactions between the carbonyl groups from NAP and CA molecules $[29,36,37]$. Moreover, on the FTIR spectrum of NAP, the strong broad absorption peak at around $3150 \mathrm{~cm}^{-1}$ is from the hydroxyl groups of NAP dimers as indicated in Figure 6. This peak disappeared in both hybrid nanofibers. 


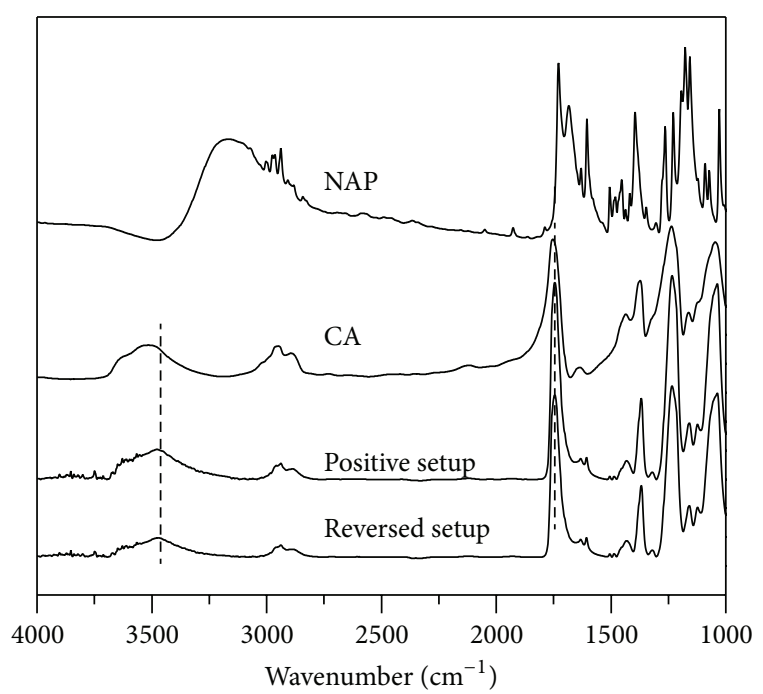

FIGURE 5: FTIR spectra of NAP-loaded nanofibers and correlated raw materials.

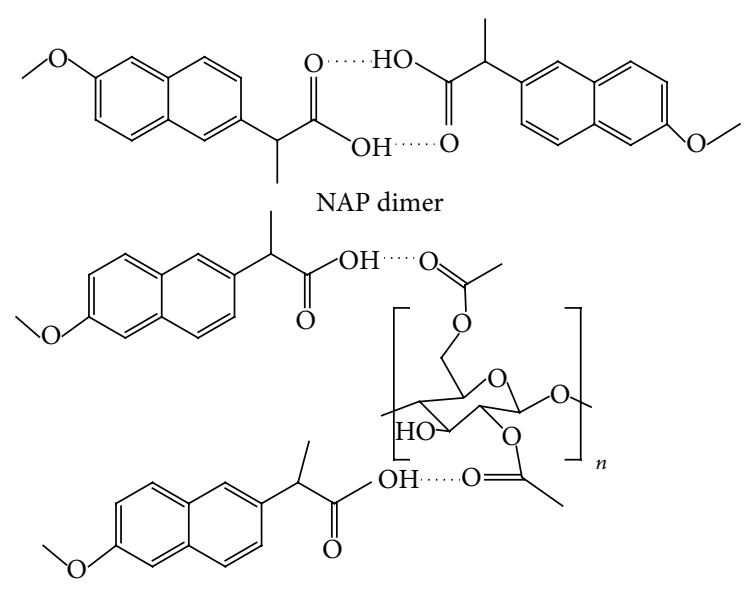

Hydrogen bonds between NAP and CA

FIGURE 6: The hydrogen bonding of NAP dimer and NAP/CA.

Meanwhile, the absorption peak from the $-\mathrm{OH}$ groups in $\mathrm{CA}$ at around $3520 \mathrm{~cm}^{-1}$ red shifts to around $3470 \mathrm{~cm}^{-1}$ due to the formation of hydrogen bonds between NAP and CA molecules. Above results confirm that NAP mixed with CA in molecular level in the resultant NAP/CA hybrid nanofibers. The interactions between NAP and CA molecules can efficiently prevent the crystallization of NAP, which is helpful for the enhancement of the solubility of NAP.

3.3. Distribution of NAP in the CA Matrix. NAP contents in the hybrid nanofibers prepared by positive and reversed emitting electrodes electrospinning setups are similar to each other. The actual NAP content is $8.89 \mathrm{wt} \%$ and $8.94 \mathrm{wt} \%$ for nanofibers prepared by positive and reversed emitting electrodes electrospinning setups, respectively. The data are similar to the feeding NAP content in the NAP/CA solution ( $9.09 \mathrm{wt} \%)$, indicating that there is no NAP loss during the

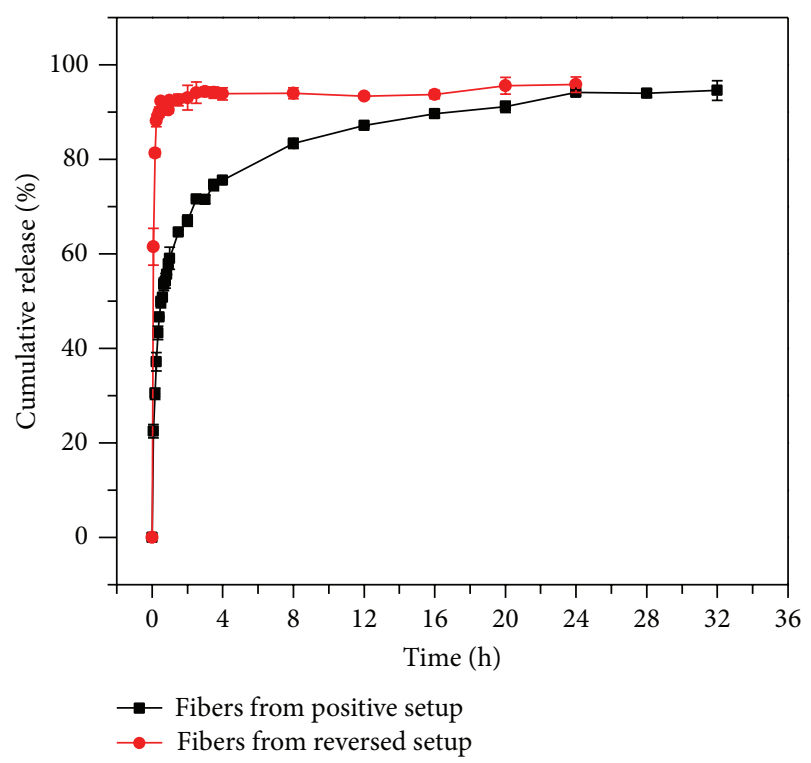

FIGURE 7: In vitro NAP release of the NAP/CA hybrid nanofibers prepared by positive and reversed emitting electrodes electrospinning setups.

preparation of the nanofibers. The in vitro NAP release profiles of the nanofibers are shown in Figure 7. Three parallel experiments were carried out and the average data and the error bar are depicted. The results showed that the NAP release of the nanofibers prepared by reversed emitting electrode electrospinning setup is much faster than that of the nanofibers prepared by the positive emitting electrode electrospinning setup (Figure 2). As reported in the literatures, the release rate of the same drug from electrospun nanofibers of the same matrix depends on the diameter of the nanofibers. The drug releasing rate of the nanofibers with thicker diameter is slower than that of the nanofibers with thinner diameter $[29,36,37]$. However, in the present work, the drug releasing rate of the NAP/CA nanofibers (diameter of $574.4 \pm 212.2 \mathrm{~nm}$ ) prepared from the positive emitting electrode approach is slower than that of the nanofibers (diameter of $594.6 \pm 197.2 \mathrm{~nm}$ ) prepared from the reversed emitting electrode approach (Figure 7), which is different from the literatures in $[29,36,37]$. The difference in the NAP release behaviors of the NAP/CA hybrid nanofibers suggests that the distribution of NAP in the nanofibers prepared by the positive and reversed emitting electrodes electrospinning approaches could be different. The fast NAP release of the nanofibers prepared by reversed emitting electrode electrospinning setup could be attributed to the fact that the NAP molecules were enriched near the surface of the nanofibers during the electrospinning. In order to approve the difference in the content of NAP near the surface of the nanofibers, EDS experiments were carried out and the data are listed in Table 1. It was found that the oxygen content in fibers prepared from the positive emitting electrode is higher than that in the nanofibers prepared from reversed approach. Considering that EDS provides exact element compositions of the nanofiber surface within depth of 5-10 $\mathrm{nm}$ and that the 
<smiles>COc1ccc2cc(C(C)C(=O)O)ccc2c1</smiles>

FIGURE 8: The ionization of NAP in the solution.

TABLE 1: Element $\mathrm{C}$ and $\mathrm{O}$ in different fibers and the raw materials.

\begin{tabular}{lcc}
\hline Materials & $\mathrm{C} / \mathrm{wt} \%$ & $\mathrm{O} / \mathrm{wt} \%$ \\
\hline $\mathrm{CA}^{*}$ & 51.72 & 4828 \\
NAP $^{*}$ & 77.78 & 22.22 \\
Fibers from positive setup & 67.92 & 32.08 \\
Fibers from reversed setup & 80.34 & 19.66 \\
\hline
\end{tabular}

${ }^{*}$ The weight fraction was calculated from its molecular formula.

oxygen content in CA is higher than that in NAP (Table 1), the EDS results suggested that NAP was enriched near the surface of the nanofibers prepared from the reversed emitting electrode, which is consistent with the conclusion derived from the NAP release profile of both nanofibers.

NAP molecules have carboxyl groups and could be ionized in CA/acetone/DMAc solution (Figure 8). The conductivities of the CA and NAP/CA solutions with acetone/DMAc mixed solvent are 0.765 and $0.857 \mathrm{mS} / \mathrm{m}$, respectively, confirming the ionization of NAP molecules in the mixed solution. During the electrospinning process, the electric field enhanced the dissociation and any structure capable of being ionized would experience a separation of charges [38]. With a positive emitting electrode electrospinning setup, the reversed ions will migrate to the inner surface of the needle and vice versa, resulting in the core part of the solution fluid with an excess of mobile charges that can respond to the electrostatic stress of the opposite electrode. In the case of the electrospinning of the NAP/CA solution with positive emitting electrode setup, the electron with smaller mass moves to the needle surface much faster than the proton with bigger mass in the case of reversed emitting electrode electrospinning setup (Figure 9), resulting in the positive flying jet with more net charges than the reversed jet, which is confirmed by the difference in the working electric current during the positive $(4.6-5.4 \mu \mathrm{A})$ and reversed $(0.3-0.5 \mu \mathrm{A})$ emitting electrodes electrospinning in the present work. It is widely accepted that the net charge moves to the surface of the flying jet and the drift velocity of ions inside the jet is about $0.1 \mathrm{~m} / \mathrm{s}[33,39,40]$. Therefore, the negatively charged NAP could move to the surface of the jet in the reversed emitting electrode electrospinning, which leads to the higher
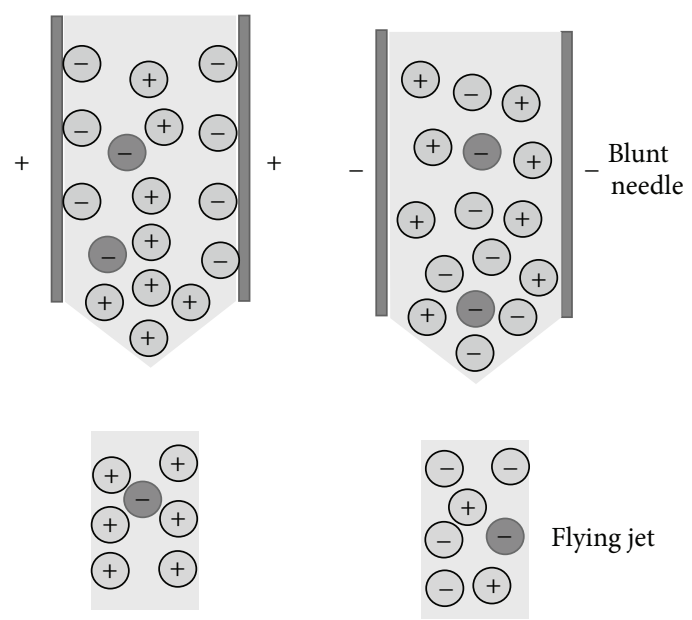

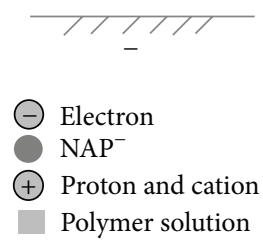

(a)

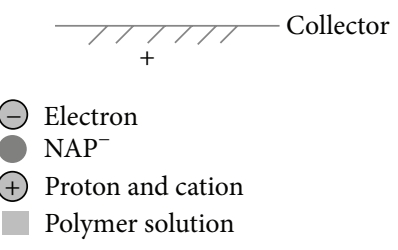

(b)
FIGURE 9: NAP distribution in the jets of (a) positive and (b) reversed emitting electrodes electrospinning setups.

NAP content near the surface of the prepared nanofibers. Therefore, the release rate of the NAP is faster from the nanofibers prepared by reversed emitting electrode electrospinning setup than from those nanofibers prepared by positive emitting electrode electrospinning setup. Considering the distribution of the loaded NAP caused by the emitting electrode polarity, drugs of different ionization properties can be embedded in the polymer matrixes of nanofibers with relatively controlled distribution by modulating the emitting electode polarity, which could be used to prepare biomedical materials for various applications, such as wound dressings and surgical scaffolds.

\section{Conclusions}

NAP/CA hybrid nanofibers were prepared by both positive and reversed emitting electrodes electrospinning setups. The nanofibers prepared by reversed emitting electrode electrospinning setup have relatively thicker diameter than those prepared by positive emitting electrode electrospinning setup. NAP is dispersed in the CA matrix in molecular level without formation of NAP aggregation and dimer. Nanofibers prepared by the reversed emitting electrode electrospinning setup have a larger amount of NAP in the surface layer, which resulted in the faster NAP release compared with the nanofibers prepared by positive emitting electrode electrospinning setup. This finding is important in producing hybrid nanofibers with drugs of different ionization property 
through blend electrospinning, which may have various drug release behaviors and then be used as biomedical materials.

\section{Supporting Information}

Nanofiber mats prepared by positive and reversed emitting electrodes electrospinning were shown in Figure S1.

\section{Conflict of Interests}

The authors declare that there is no conflict of interests regarding the publication of this paper.

\section{Acknowledgments}

This work was supported by the National Natural Science Foundation of China (nos. 21174150 and 21174156) and the Knowledge Innovation Program of the Chinese Academy of Sciences (no. KJCX2-YW-H19).

\section{References}

[1] J. Doshi and D. H. Reneker, "Electrospinning process and applications of electrospun fibers," Journal of Electrostatics, vol. 35, no. 2-3, pp. 151-160, 1995.

[2] A. Greiner and J. H. Wendorff, "Electrospinning: a fascinating method for the preparation of ultrathin fibers," Angewandte Chemie International Edition, vol. 46, no. 30, pp. 5670-5703, 2007.

[3] L. Chen, L. Bromberg, T. A. Hatton, and G. C. Rutledge, "Catalytic hydrolysis of p-nitrophenyl acetate by electrospun polyacrylamidoxime nanofibers," Polymer, vol. 48, no. 16, pp. 4675-4682, 2007.

[4] L. Chen, L. Bromberg, H. Schreuder-Gibson, J. Walker, T. A. Hatton, and G. C. Rutledge, "Chemical protection fabrics via surface oximation of electrospun polyacrylonitrile fiber mats," Journal of Materials Chemistry, vol. 19, no. 16, pp. 2432-2438, 2009.

[5] J. Shi, L. Wang, F. Zhang et al., "Incorporating protein gradient into electrospun nanofibers as scaffolds for tissue engineering," ACS Applied Materials \& Interfaces, vol. 2, no. 4, pp. 1025-1030, 2010.

[6] Y. Tian, M. Wu, R. Liu et al., "Electrospun membrane of cellulose acetate for heavy metal ion adsorption in water treatment," Carbohydrate Polymers, vol. 83, no. 2, pp. 743-748, 2011.

[7] H. Kang, Y. Zhu, X. Yang, Y. Jing, A. Lengalova, and C. $\mathrm{Li}$, "A novel catalyst based on electrospun silver-doped silica fibers with ribbon morphology," Journal of Colloid and Interface Science, vol. 341, no. 2, pp. 303-310, 2010.

[8] Z. Zhang, C. Shao, Y. Sun et al., "Tubular nanocomposite catalysts based on size-controlled and highly dispersed silver nanoparticles assembled on electrospun silica nanotubes for catalytic reduction of 4-nitrophenol," Journal of Materials Chemistry, vol. 22, no. 4, pp. 1387-1395, 2012.

[9] Y. Huang, H. Ma, S. Wang et al., "Efficient catalytic reduction of hexavalent chromium using palladium nanoparticleimmobilized electrospun polymer nanofibers," ACS Applied Materials \& Interfaces, vol. 4, no. 6, pp. 3054-3061, 2012.
[10] Y. Long, H. Chen, Y. Yang et al., "Electrospun nanofibrous film doped with a conjugated polymer for DNT fluorescence sensor," Macromolecules, vol. 42, no. 17, pp. 6501-6509, 2009.

[11] X. W. Mao, F. Simeon, G. C. Rutledge, and T. A. Hatton, "Electrospun carbon nanofiber webs with controlled density of states for sensor applications," Advanced Materials, vol. 25, no. 9, pp. 1309-1314, 2013.

[12] G. D. Fu, L. Q. Xu, F. Yao et al., "Smart nanofibers from combined living radical polymerization, "click chemistry", and electrospinning," ACS Applied Materials \& Interfaces, vol. 1, no. 2, pp. 239-243, 2009.

[13] H.-J. Jin, S. V. Fridrikh, G. C. Rutledge, and D. L. Kaplan, "Electrospinning Bombyx mori silk with poly(ethylene oxide)," Biomacromolecules, vol. 3, no. 6, pp. 1233-1239, 2002.

[14] M. Wang, H.-J. Jin, D. L. Kaplan, and G. C. Rutledge, "Mechanical properties of electrospun silk fibers," Macromolecules, vol. 37, no. 18, pp. 6856-6864, 2004.

[15] H. Bai, J. Ju, Y. Zheng, and L. Jiang, "Functional fibers with unique wettability inspired by spider silks," Advanced Materials, vol. 24, no. 20, pp. 2786-2791, 2012.

[16] M. M. Hohman, M. Shin, G. Rutledge, and M. P. Brenner, "Electrospinning and electrically forced jets. II. Applications," Physics of Fluids, vol. 13, no. 8, pp. 2221-2236, 2001.

[17] M. M. Hohman, M. Shin, G. Rutledge, and M. P. Brenner, "Electrospinning and electrically forced jets. I. Stability theory," Physics of Fluids, vol. 13, no. 8, pp. 2201-2220, 2001.

[18] S. Sukigara, M. Gandhi, J. Ayutsede, M. Micklus, and F. Ko, "Regeneration of Bombyx mori silk by electrospinning-part 1: processing parameters and geometric properties," Polymer, vol. 44, no. 19, pp. 5721-5727, 2003.

[19] S.-H. Tan, R. Inai, M. Kotaki, and S. Ramakrishna, "Systematic parameter study for ultra-fine fiber fabrication via electrospinning process," Polymer, vol. 46, no. 16, pp. 6128-6134, 2005.

[20] C. J. Thompson, G. G. Chase, A. L. Yarin, and D. H. Reneker, "Effects of parameters on nanofiber diameter determined from electrospinning model," Polymer, vol. 48, no. 23, pp. 6913-6922, 2007.

[21] M. Chowdhury and G. K. Stylios, "Analysis of the effect of experimental parameters on the morphology of electrospun polyethylene oxide nanofibres and on their thermal properties," Journal of the Textile Institute, vol. 103, no. 2, pp. 124-138, 2012.

[22] A. Kilic, F. Oruc, and A. Demir, "Effects of polarity on electrospinning process," Textile Research Journal, vol. 78, no. 6, pp. 532-539, 2008.

[23] P. Supaphol, C. Mit-Uppatham, and M. Nithitanakul, "Ultrafine electrospun polyamide- 6 fibers: effect of emitting electrode polarity on morphology and average fiber diameter," Journal of Polymer Science B: Polymer Physics, vol. 43, no. 24, pp. 36993712, 2005.

[24] U. Ali, X. Wang, and T. Lin, "Effect of nozzle polarity and connection on electrospinning of polyacrylonitrile nanofibers," Journal of the Textile Institute, vol. 103, no. 11, pp. 1160-1168, 2012.

[25] J. Lin, C. Li, Y. Zhao, J. Hu, and L.-M. Zhang, "Co-electrospun nanofibrous membranes of collagen and zein for wound healing," ACS Applied Materials \& Interfaces, vol. 4, no. 2, pp. 10501057, 2012.

[26] C. Tang, A. E. Ozcam, B. Stout, and S. A. Khan, "Effect of $\mathrm{pH}$ on protein distribution in electrospun PVA/BSA composite nanofibers," Biomacromolecules, vol. 13, no. 5, pp. 1269-1278, 2012. 
[27] R. A. Thakur, C. A. Florek, J. Kohn, and B. B. Michniak, "Electrospun nanofibrous polymeric scaffold with targeted drug release profiles for potential application as wound dressing," International Journal of Pharmaceutics, vol. 364, no. 1, pp. 8793, 2008.

[28] S. Tungprapa, I. Jangchud, and P. Supaphol, "Release characteristics of four model drugs from drug-loaded electrospun cellulose acetate fiber mats," Polymer, vol. 48, no. 17, pp. 50305041, 2007.

[29] D.-G. Yu, X.-Y. Li, X. Wang, W. Chian, Y.-Z. Liao, and Y. $\mathrm{Li}$, "Zero-order drug release acetate nanofibers prepared using coaxial electrospinning," Cellulose, vol. 20, no. 1, pp. 379-389, 2012.

[30] C. Derry, S. Derry, R. A. Moore, and H. J. McQuay, "Single dose oral naproxen and naproxen sodium for acute postoperative pain in adults," Cochrane Database of Systematic Reviews, no. 1, Article ID CD004234, 2009.

[31] M.-N. Giraud, C. Motta, J. J. Romero, G. Bommelaer, and L. M. Lichtenberger, "Interaction of indomethacin and naproxen with gastric surface-active phospholipids: a possible mechanism for the gastric toxicity of nonsteroidal anti-inflammatory drugs (NSAIDs)," Biochemical Pharmacology, vol. 57, no. 3, pp. 247$254,1999$.

[32] K. E. S. Locock, T. D. Michl, J. D. P. Valentin et al., "Guanylated polymethacrylates: a class of potent antimicrobial polymers with low hemolytic activity," Biomacromolecules, vol. 14, no. 11, pp. 4021-4031, 2013.

[33] Y. M. Shin, M. M. Hohman, M. P. Brenner, and G. C. Rutledge, "Experimental characterization of electrospinning: the electrically forced jet and instabilities," Polymer, vol. 42, no. 25, pp. 9955-9967, 2001.

[34] S. A. Theron, E. Zussman, and A. L. Yarin, "Experimental investigation of the governing parameters in the electrospinning of polymer solutions," Polymer, vol. 45, no. 6, pp. 2017-2030, 2004.

[35] W.-E. Teo, R. Inai, and S. Ramakrishna, "Technological advances in electrospinning of nanofibers," Science and Technology of Advanced Materials, vol. 12, no. 1, Article ID 013002, 2011.

[36] D. G. Yu, X. Wang, X. Y. Li, W. Chian, Y. Li, and Y. Z. Liao, "Electrospun biphasic drug release polyvinylpyrrolidone/ethyl cellulose core/sheath nanofibers," Acta Biomaterialia, vol. 9, no. 3, pp. 5665-5672, 2013.

[37] D.-G. Yu, J.-H. Yu, L. Chen, G.-R. Williams, and X. Wang, "Modified coaxial electrospinning for the preparation of highquality ketoprofen-loaded cellulose acetate nanofibers," Carbohydrate Polymers, vol. 90, no. 2, pp. 1016-1023, 2012.

[38] G. Collins, J. Federici, Y. Imura, and L. H. Catalani, "Charge generation, charge transport, and residual charge in the electrospinning of polymers: a review of issues and complications," Journal of Applied Physics, vol. 111, no. 4, Article ID 044701, 2012.

[39] D. H. Reneker, A. L. Yarin, H. Fong, and S. Koombhongse, "Bending instability of electrically charged liquid jets of polymer solutions in electrospinning," Journal of Applied Physics, vol. 87, no. 9 I, pp. 4531-4547, 2000.

[40] Z. Zha, S. L. Leung, Z. Dai, and X. Wu, "Centering of organicinorganic hybrid liposomal cerasomes in electrospun gelatin nanofibers," Applied Physics Letters, vol. 100, no. 3, Article ID 033702, 3 pages, 2012. 

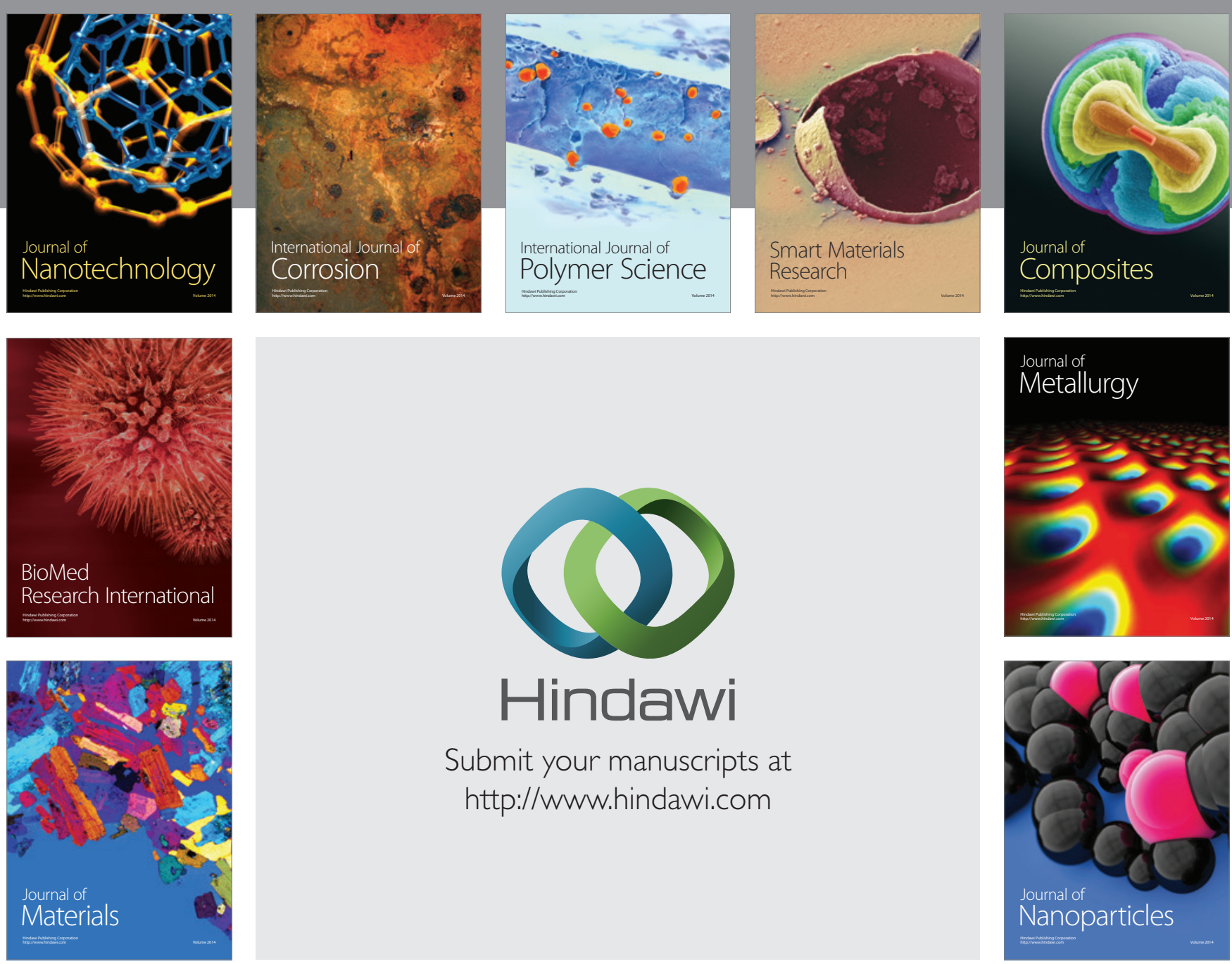

Submit your manuscripts at http://www.hindawi.com
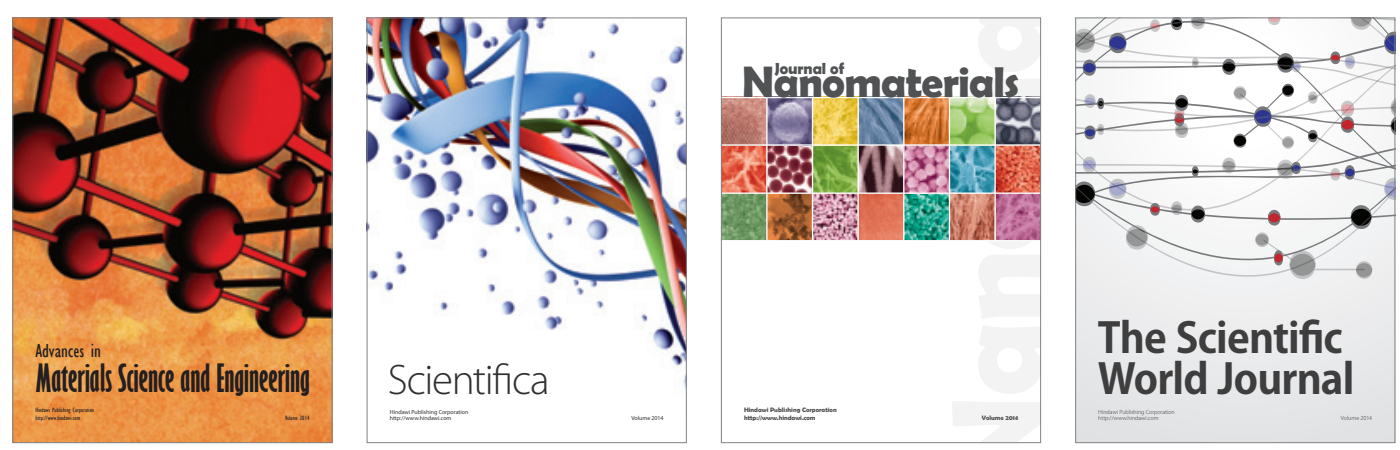

\section{The Scientific World Journal}
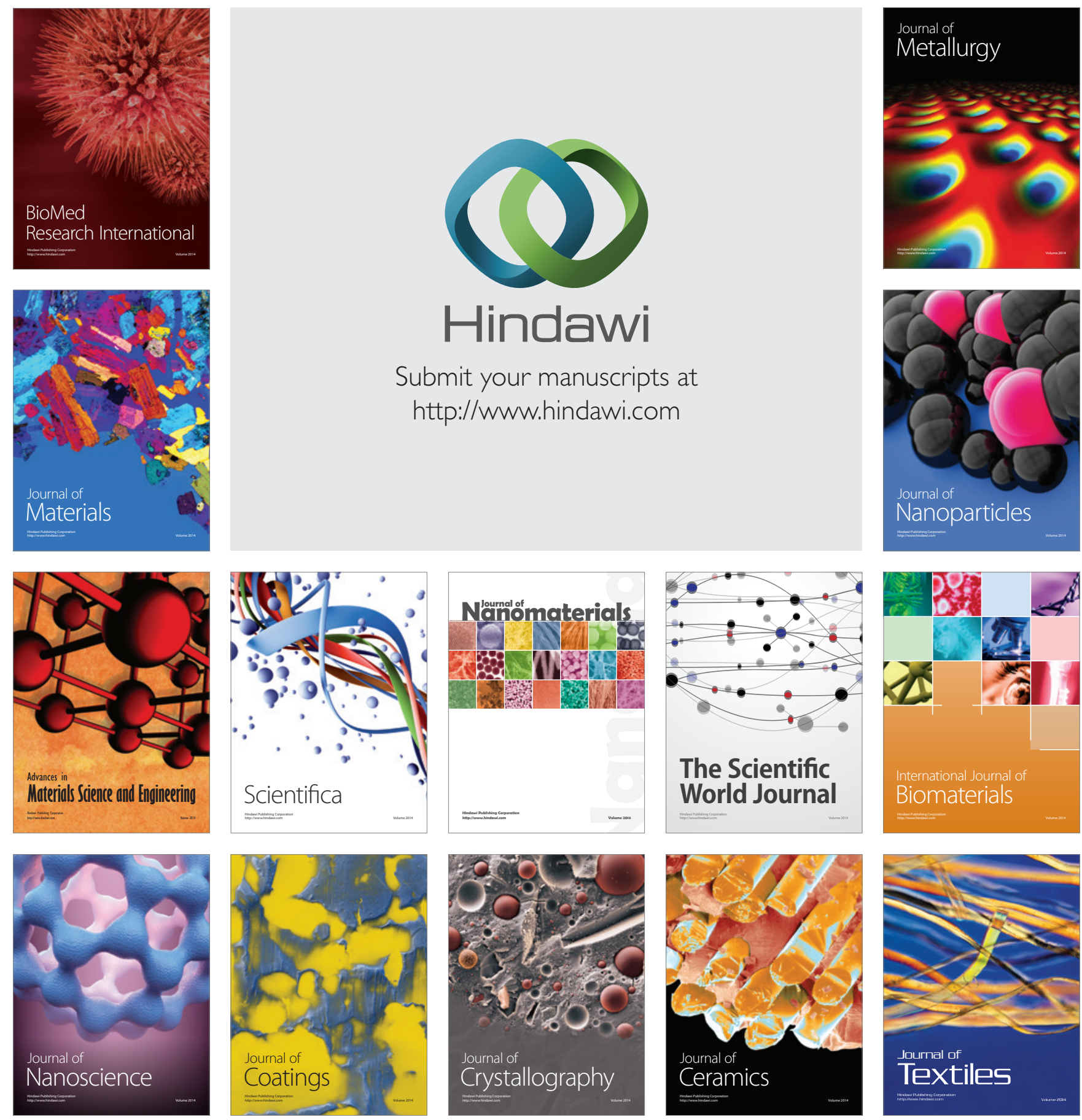